ing in a nonlinear complex system driven far from equilibrium (25). In this case, the model suggests that essentially all of the carbon condensing on the cathode is initially in the form of perfect fullerene fibers, purified in the "zone refining" of the arc. Although these nanofibers are highly stable in isolation on the nanometer scale, when touching each other on the macroscale they are, unfortunately, only metastable. The same fire that gives birth to them in the arc also leads to their destruction by sintering (26). Still, much can be made from those nanotubes that survive (imperfect as they may be) in the ashes of zone 1 and in the tangles of zone 2 .

\section{REFERENCES AND NOTES}

1. S. lijima, Nature 354, 56 (1991).

2. T. W. Ebbesen and P. M. Ajayan, ibid. 358, 220 (1992).

3. K. Tanaka et al., Chem. Phys. Lett. 223, 65 (1994).

4. T. W. Ebbesen, P. M. Ajayan, H. Hiura, K. Tanigaki, Nature 367, 519 (1994).

5. O. Zhou et al., Science 263, 1744 (1994).

6. R. E. Haufler et al., Mater. Res. Soc. Symp. Proc. 206, 627 (1991).

7. X. K. Wang, X. W. Lin, V. P. Dravid, J. B. Ketterson, R. P. H. Chang, Appl. Phys. Lett. 62, 1881 (1993).

8. Y. Chai et al., J. Phys. Chem. 95, 7564 (1991).

9. C. Yeretzian, K. Hansen, F. Diederich, R. L. Whetten, Nature 359, 44 (1992)

10. R. D. Beck, P. Weis, G. Brauchle, M. M. Kappes, J. Chem. Phys. 100, 262 (1994).

11. T. W. Ebbesen, H. Hiura, Y. Ochiai, S. Matsui, K. Tanigaki, Chem. Phys. Lett. 209, 83 (1993).

12. Y. Saito, T. Yoshikawa, M. Inagaki, M. Tomita, T. Hayashi, ibid. 204, 277 (1993).

13. S. Seraphin, D. Zhou, J. Jiao, Carbon 31, 1212 (1993).

14. The arcing power supply was an arc welder (Hobart "Porta Tig/Stick," Troy, OH) operating under constant current control, filtered to a residual 3\% peakto-peak sinusoidal ripple at $12 \mathrm{kHz}$ when operating at 100 to $300 \mathrm{~A}$ between 15 and $25 \mathrm{~V}$. During operation, the voltage drop across the arc was monitored and via a feedback loop; the vertical separation of the electrodes was adjusted to maintain the desired operating voltage.

15. The five-membered rings (pentagons) that give onionlike particles and closed tube caps their curvature are susceptible to oxidation, in contrast to the perfect graphene tube walls, which are impervious to such oxidative attack. The vastly smaller relative surface area susceptible to etching of long nanotubes compared with their shorter cousins allows the former to outlast the latter under oxidative conditions. This behavior is a manifestation of the one-dimensional nature of carbon nanotubes.

16. R. E. Smalley, Mater. Sci. Eng. B19, 1 (1993).

17. T. W. Ebbesen, Annu. Rev. Mater. Sci. 24, 235 (1994).

18. Although the conformation with the tube tip open has a higher energy than the closed conformation even in the presence of an electric field, the concentration of negative charge at the tip under such conditions leads to a dramatic increase in the kinetic barrier to closure (L. Luo, P. Nordlander, R. E. Smalley, unpublished results).

19. See, for example, Y. P. Raizer, Gas Discharge Physics (Springer-Verlag, Berlin, 1991), chap. 10, and references therein.

20. We estimate the degree of ionization by calculating the carbon cation and neutral densities as follows. The cation density is $n_{+}=j_{+} / v_{+}$, where $j_{+}$is the cation current density and $v_{+}$is its drift velocity. The cations carry $\sim 20 \%$ of the total current [compare with (19)], thus $j_{+} \simeq 40 \mathrm{Avcm}^{2}$. (We have used here only the area belonging to zone 1.) We estimate the drift velocity to be $\sim 10^{4} \mathrm{~cm} \mathrm{~s}^{-1}$, yielding a cation density of $\sim 2.5 \times 10^{16} \mathrm{~cm}^{-3}$. Because the mass of carbon soot lost from the plasma is only $\sim 10 \%$ that of the deposit, we can calculate the carbon neutral density as the flux of atoms lost to the chamber divided by their mean velocity, yielding $2 \times 10^{15} \mathrm{~cm}^{-3}$. Thus, we find an unusually high degree of ionization of $\sim 90 \%$, corresponding to a highly nonequilibrium plasma, contrasting with equilibrium carbon plasmas, which have only a few percent of carbon atoms ionized.

21. A. Yabe, Y. Mori, K. Hijikata, AIAA J. 16, 340 (1978).

22. E. L. Koschmieder, Bénard Cells and Taylor Vortices (Cambridge Univ. Press, New York, 1993).

23. We have established that the direction of gravity has no effect on the formation of zones 1 and 2. The same pattern is found on the cathode deposit regardless of whether it is the cathode or the anode that is "on top."

24. If we assume the bulk of the current flows to the tops of the zone 1 columns, the current density there is $\sim 500 \mathrm{~A} \mathrm{~cm}^{-2}$, which corresponds to $3 \times 10^{20} \mathrm{car}$ bon ions per second per square centimeter, if we assume that $80 \%$ of the current is carried by the electrons.

25. See, for example, R. Ruthen, Sci. Am. 268, 130 (January 1993); B. Kaye, Chaos and Complexity (VCH Press, New York, 1993); F. Cramer, Chaos and Order (VCH Press, New York, 1993), and references therein.

26. This research was supported by the Office of Naval Research, the National Science Foundation, and the Robert A. Welch Foundation, and used equipment designed for study of fullerene-encapsulated catalysts supported by the Department of Energy, Division of Chemical Sciences.

\title{
A Phase of Liposomes with Entangled Tubular Vesicles
}

\section{Shivkumar Chiruvolu, Heidi E. Warriner, Edward Naranjo, Stefan H. J. Idziak, Joachim O. Rädler, Robert J. Plano, Joseph A. Zasadzinski, ${ }^{\star}$ Cyrus R. Safinya*}

An equilibrium phase belonging to the family of bilayer liposomes in ternary mixtures of dimyristoylphosphatidylcholine (DMPC), water, and geraniol (a biological alcohol derived from oil-soluble vitamins that acts as a cosurfactant) has been identified. Electron and optical microscopy reveal the phase, labeled $L_{t v}$, to be composed of highly entangled tubular vesicles. In situ $x$-ray diffraction confirms that the tubule walls are multilamellar with the lipids in the chain-melted state. Macroscopic observations show that the $L_{\text {tv }}$ phase coexists with the well-known $L_{4}$ phase of spherical vesicles and a bulk $L_{\alpha}$ phase. However, the defining characteristic of the $L_{t v}$ phase is the Weissenberg rod climbing effect under shear, which results from its polymer-like entangled microstructure.

Phospholipid molecules form closed bilayer shells known as liposomes or vesicles when dispersed in water because of the amphiphilic nature of the molecules (1). Since their discovery by Bangham et al. (2), uni- and multilamellar vesicles have received much attention because of their similarities to living cells and their potential for encapsulating and segregating water-soluble materials from a bulk solution (1). Vesicles are used extensively as models of adhesion, de-adhesion, and fusion of interacting cells $(1,3)$

S. Chiruvolu and E. Naranjo, Materials Research Laboratory and Department of Chemical Engineering, University of California, Santa Barbara, CA 93106, USA

H. E. Warriner, Materials Research Laboratory, Materials Department, and Physics Department, University of California, Santa Barbara, CA 93106, USA

S. H. J. Idziak and J. O. Rädler, Materials Research Laboratory and Materials Department, University of California, Santa Barbara, CA 93106, USA.

R. J. Plano, Exxon Research and Engineering Company, Annandale, NJ 08801, USA.

J. A. Zasadzinski, Materials Research Laboratory, De partment of Chemical Engineering, and Materials Department, University of California, Santa Barbara, CA 93106, USA.

C. R. Safinya, Materials Research Laboratory, Materials Department, Physics Department, and Biochemistry and Molecular Biology Program, University of California, Santa Barbara, CA 93106, USA

*To whom correspondence should be addressed. and in fundamental studies of colloidal interactions and stability $(4,5)$. From a technological viewpoint, vesicles are increasingly used in the cosmetics industries as controlled chemical release agents (such as in formulations of lotions, gels, and creams) (6); they continue to be explored for their utility in the food and agricultural industries and are likely to dramatically impact the medical field as drug and gene carriers (7).

We report on the discovery of another vesicle phase in room-temperature mixtures of DMPC, water, and geraniol. Geraniol is a branched long-chain biological alcohol, derived from oil-soluble vitamins, that acts as a cosurfactant (Fig. 1). In the DMPC-rich corner of the phase diagram, we observe a lyotropic liquid-crystal multilamellar $\mathrm{L}_{\alpha}$ phase, and in the water-rich corner, an extremely dilute phase of spherical unilamellar vesicles that is similar to the equilibrium vesicle phases $\left(\mathrm{L}_{4}\right)$ recently observed in similar systems (8-10). A phase consisting of entangled multilamellar tubular vesicles, which we have labeled the $L_{t v}$ phase, emerges in the region between the $\mathrm{L}_{\alpha}$ and $\mathrm{L}_{4}$ phases. The $\mathrm{L}_{\mathrm{tv}}$ phase responds dramatically to applied flow fields, which strongly indicates that its microstructure is similar to that of a semidi- 
lute, entangled polymer solution. Typical dilute uni- and multilamellar vesicles cannot become entangled because of their closed, spherical topology and hence have rather simple, solvent-dominated behavior under shear (10).

Myelin-like cylindrical vesicles similar to the $L_{t v}$ phase have been observed in the early stages of hydration of lecithin samples (11). However, the formation of these heterogeneous samples depended on the method of lipid hydration and were primarily made up of large spherical multilamellar vesicles; the nonspherical structures gradually evolved into spherical structures on equilibration. Here we report on a distinct situation associated with the equilibrium phase behavior of a ternary system. Upon simply mixing DMPC, geraniol, and water at the appropriate ratios and storing the solution at $25^{\circ} \mathrm{C}$ without agitation, tubular vesicles form spontaneously. Over a wide variety of concentrations, long-term phase coexistence is observed, either between the $L_{t v}$ and $\mathrm{L}_{4}$ phases or the $\mathrm{L}_{\alpha}$ and $\mathrm{L}_{\mathrm{tv}}$ phases (Figs. 1 and 2).

A key ingredient leading to the formation of this new phase is the presence of an alcohol or "cosurfactant." Cosurfactants, such as alcohols, act in a fashion similar to that of surfactants in that they partition preferentially into the bilayer, although they typically do not undergo extensive self-assembly. Alcohol cosurfactants are known to qualitatively alter phase behavior when present as a majority component in the bilayer, typically two to four cosurfactant molecules per surfactant (12-15). The stiffness or bending rigidity $\left(k_{\mathrm{c}} \approx 50 k_{\mathrm{B}} T\right.$, where $k_{\mathrm{B}}$ is Boltzmann's constant and $T$ is temperature) of DMPC-water multilayers in the $\mathrm{L}_{\alpha}$ phase $(16,17)$ may be strongly reduced by the addition of the cosurfactant pentanol (18), which decreases the bilayer thickness and makes the membranes more flexible. As a result, an enhanced undulation repulsion $(15,18-21)$ between mixed lipid-cosurfactant bilayers enables dilution of the stacked membrane phase to interlayer spacings much larger than the membrane thickness [which is typical of rigid membranes $(4,22)]$.

The ternary phase diagram of DMPCgeraniol-water system at $25^{\circ} \mathrm{C}$ (Fig. 1) has four single-phase domains along with regions (shaded) where two or more of these phases coexist. Single-phase domains occur in each of the DMPC-, water-, and geraniol-rich corners of the phase diagram; they are, respectively, the well-known $L_{\alpha}$ or lamellar phase, an extremely dilute vesicle phase $\left(\mathrm{L}_{4}\right)$, and a reverse-micellar phase. In binary DMPC-water mixtures, at concentrations less than about 40 weight $\%$ water, an $\mathrm{L}_{\alpha}$ phase is formed (22); however, in ternary DMPCgeraniol-water mixtures at $\sim 14$ weight $\%$ geraniol, the $\mathrm{L}_{\alpha}$ phase can be diluted to $\sim 46$ weight \% water (resulting in an interlayer spacing $d=63 \AA$ ). At about the same ratio

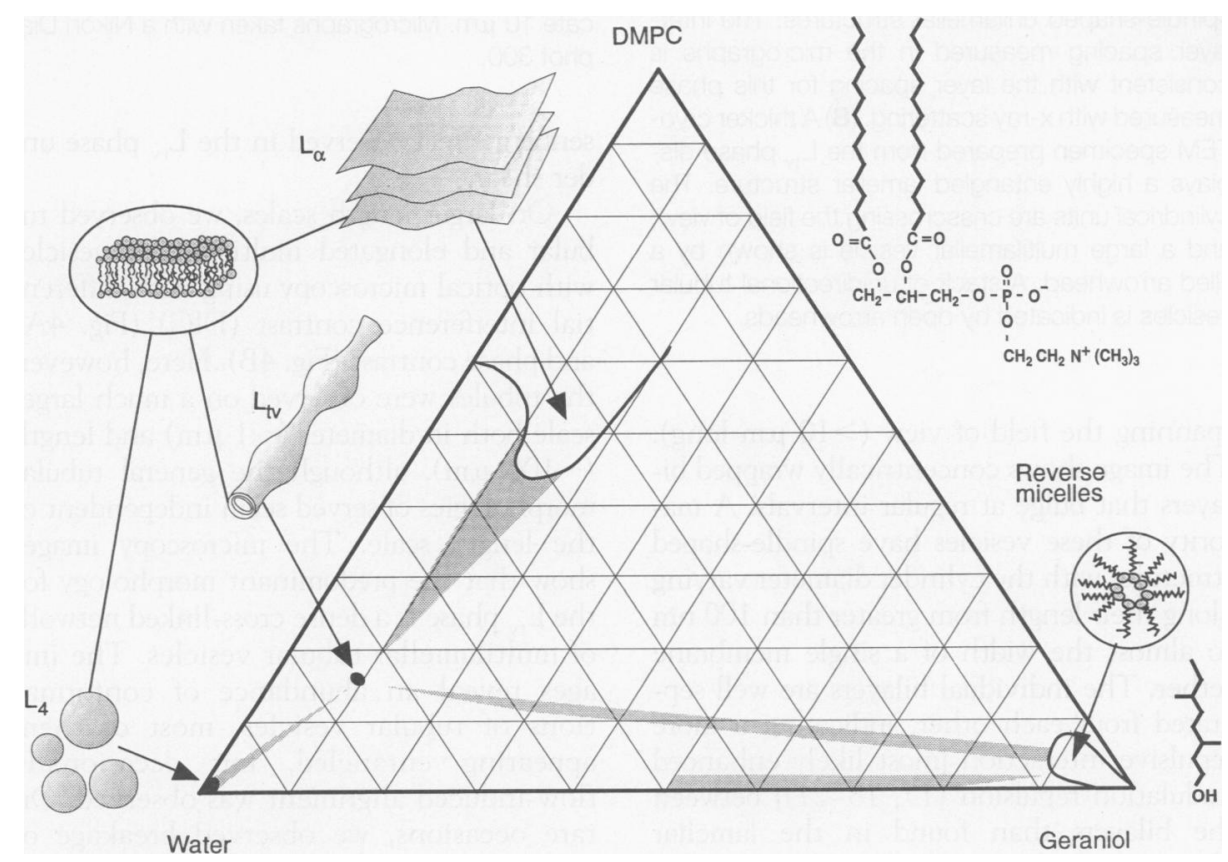

Fig. 1. Temary phase diagram of DMPC-geraniol-water at $25^{\circ} \mathrm{C}[\mathrm{DMPC}$ and geraniol were purchased from Avanti Polar Lipids (Alabaster, Alabama) and Sigma (St. Louis, Missouri), respectively]. The two-phase regions are shown as shaded areas and the one-phase lamellar $\left(L_{\alpha}\right)$ and reverse-micellar phases are outlined by solid lines. Probable single-phase regions of the tubular $L_{v}$ and the unilamellar spherical $L_{4}$ phase vesicles are indicated by black dots because they were found only in restricted regimes of concentration. The structure at the top right is DMPC, a double-tailed bilayer-forming lipid, and the structure at the bottom right is geraniol, a branched biological alcohol. of DMPC to geraniol, dilution of the lamellar phase to greater than 46 weight $\%$ water leads to phase separation, giving rise to a bluish and transparent $L_{t v}$ phase on the surface that coexists with a lamellar $L_{\alpha}$ phase that settles to the bottom of the sample vial. In Fig. 1, the shaded area below the $\mathrm{L}_{\alpha}$ phase is a two-phase region where the $\mathrm{L}_{\mathrm{tv}}$ phase coexists with the lamellar phase.

When samples are prepared at approximately 75 weight \% water, 15 weight \% DMPC, and 10 weight \% geraniol, the predominant phase formed ( $>90 \%$ by volume) is the bluish $\mathrm{L}_{\mathrm{tv}}$ phase. The $\mathrm{L}_{\mathrm{tv}}$ phase appears to be an equilibrium phase; sealed solutions of this phase have remained stable for more than 2 years. The new phase is stable at $25^{\circ} \mathrm{C}$, appearing isotropic between cross-polarizers, and is temperature-
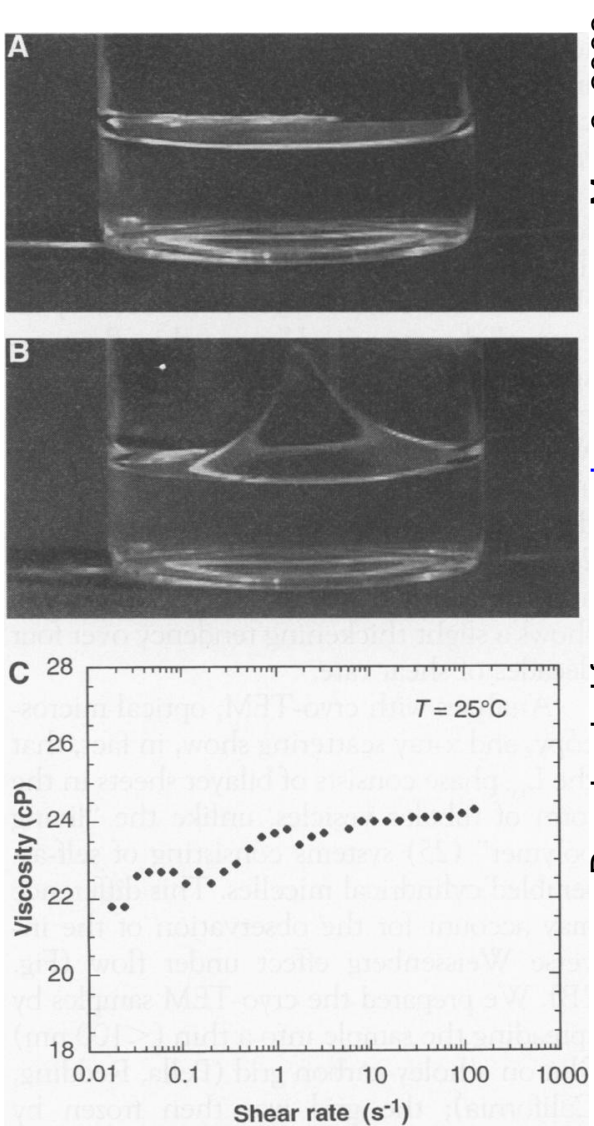

Fig. 2. (A) Photograph of a test tube showing the interface of a two-phase sample with a tubular vesicle phase $L_{t v}$ (top) and a dilute $L_{4}$ spherical vesicle phase (bottom). (B) When a rotating rod (not shown) is inserted into the upper phase, the observed response is characteristic of a densely entangled semidilute polymer liquid: The interface moves inward and upward as described in the text. (C) Viscosity of the $L_{t v}$ phase $(80$ weight $\%$ water, 14 weight $\%$ DMPC, and 6 weight $\%$ geraniol) in centipoise (1 $\mathrm{P}=1$ dyne. $\left.\mathrm{s} \mathrm{cm}^{-2}\right)$ plotted versus the shear rate at $25^{\circ} \mathrm{C}$. The viscous phase shows slight thickening over the four decades of measured shear rate. The instrument used was a lowshear rotational couette rheometer (Contraves LS30, AG Zürich, Switzerland). 
sensitive, turning cloudy upon cooling.

Particularly interesting is the flow behavior at the interface of the $\mathrm{L}_{\mathrm{tv}}$ and $\mathrm{L}_{4}$ phases. We made a mixture of 76 weight $\%$ water, 18 weight $\%$ DMPC, and 6 weight $\%$ geraniol with a well-defined interface (Fig. 2A). Cryogenic transmission electron microscopy (cryo-TEM) has shown that the bottom phase consists of dilute spherical vesicles $\left(\mathrm{L}_{4}\right)$ (Fig. 1) and that the upper phase is the $\mathrm{L}_{\mathrm{tv}}$ phase. The top phase shows rather dramatic flow effects (Fig. 2B); a rotating rod inserted into the top phase leads to an immediate response in which the interface moves inward and climbs toward the rod at the center. This behavior, although uncommon in typical vesicle dispersions, is reminiscent of the so-called Weissenberg effect (23) observed in semidilute polymer solutions. We note, however, that the $L_{t v}$ phase exhibits an "inverse" Weissenberg effect; the typical Weissenberg effect would lead to the interface moving inward and dipping at the center. This inverted behavior may arise from a larger second normal stress difference of the $L_{t v}$ phase under flow than is usually found in semidilute polymeric solutions (24). The Weissenberg effect is a consequence of flow-induced normal stresses induced by an entangled network. Hence, this flow response of the $L_{t v}$ phase is strongly suggestive of a highly entangled microstructure. The Weissenberg effect is the most distinctive and unusual signature of this phase. More detailed measurements of the $\mathrm{L}_{\mathrm{tv}}$ phase (Fig. 2C) show that it is quite viscous, between 22 and 24 times the viscosity of water, and shows a slight thickening tendency over four decades of shear rate.

Analyses with cryo-TEM, optical microscopy, and x-ray scattering show, in fact, that the $L_{t v}$ phase consists of bilayer sheets in the form of tubular vesicles, unlike the "living polymer" (25) systems consisting of self-assembled cylindrical micelles. This difference may account for the observation of the inverse Weissenberg effect under flow (Fig. 2B). We prepared the cryo-TEM samples by spreading the sample into a thin $(<100 \mathrm{~nm})$ film on a holey-carbon grid (Pella, Redding, California); the grid was then frozen by plunging it into liquid ethane cooled by liquid nitrogen. Plunging resulted in vitrification of the solvent, and the solution microstructure was preserved. We avoided evaporation losses and temperature effects by preparing the films in a temperature- and humidity-controlled environmental chamber (26). The films were examined at $-170^{\circ} \mathrm{C}$ in a JEM 2000FX (Peabody, Massachusetts) scanning transmission electron microscope with a GATAN (Pleasanton, California) cryotransfer system.

A cryo-TEM image of a relatively thin area of one such sample of $\mathrm{L}_{\mathrm{tv}}$ phase (Fig. $3 \mathrm{~A})$ reveals extended cylindrical structures
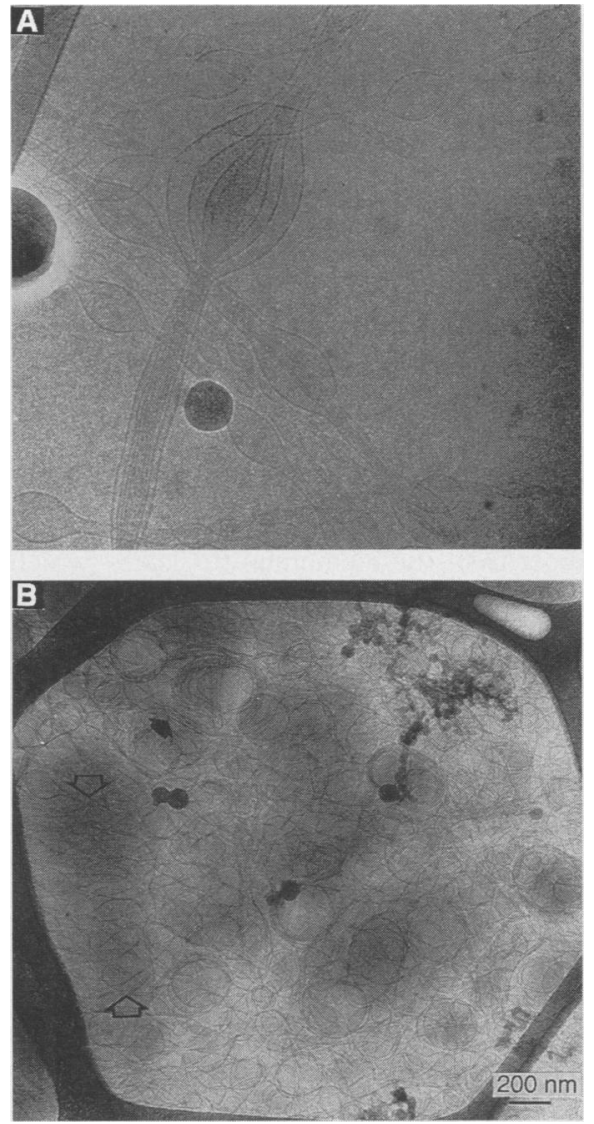

Fig. 3. (A) Cryo-TEM image of a thin section of the $L_{t v}$ phase [taken from the top of a two-phase sample $(97.4$ weight $\%$ water, 2.1 weight \% DMPC, and 0.5 weight $\%$ geraniol] at $25^{\circ} \mathrm{C}$ showing multilayered cylindrical vesicles along with spindle-shaped unilamellar structures. The interlayer spacing measured in the micrographs is consistent with the layer spacing for this phase measured with $\mathrm{x}$-ray scattering. (B) A thicker cryoTEM specimen prepared from the $L_{\mathrm{tv}}$ phase displays a highly entangled lamellar structure. The cylindrical units are crisscrossing the field of view, and a large multilamellar vesicle is shown by a filled arrowhead. A stack of unidirectional tubular vesicles is indicated by open arrowheads.

spanning the field of view (>10 $\mu \mathrm{m}$ long). The image shows concentrically wrapped bilayers that bulge at regular intervals. A majority of these vesicles have spindle-shaped structures with the cylinder diameter varying along their length from greater than $100 \mathrm{~nm}$ to almost the width of a single membrane tether. The individual bilayers are well separated from each other, indicating a more repulsive interaction [most likely enhanced undulation repulsion $(15,18-21)]$ between the bilayers than found in the lamellar phase, in which the bilayer spacing is about the same as the bilayer thickness (22). A thicker area of the cryo-TEM specimen (Fig. 3B) shows that these tubular vesicles can be highly entangled and take on a variety of spherical and cylindrical shapes. This level of entanglement is consistent with the Weis-
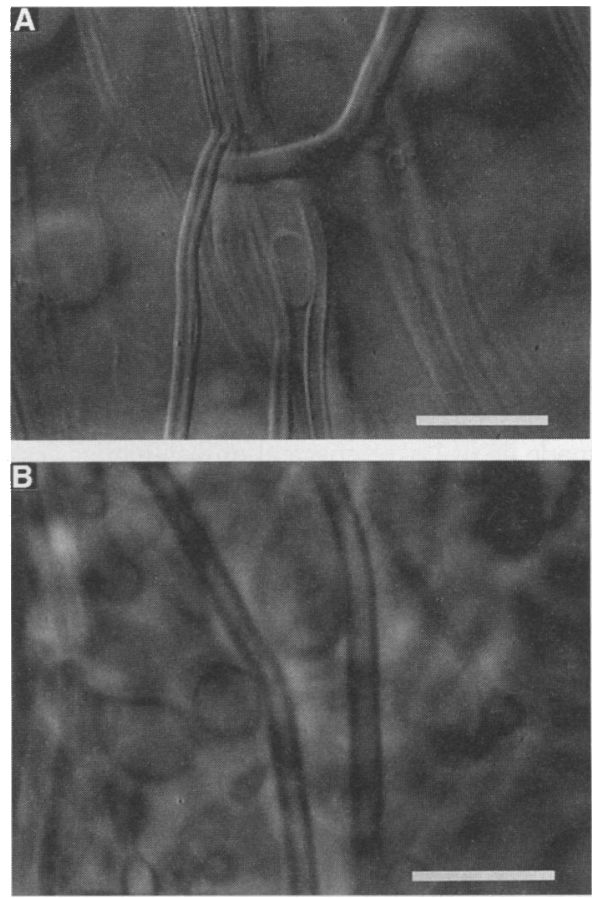

Fig. 4. (A) Optical micrograph with DIC depicting the multilayered tubular vesicles of the $L_{t v}$ phase (97.4 weight \% water, 2.1 weight \% DMPC, and 0.5 weight $\%$ geraniol at $25^{\circ} \mathrm{C}$ ) with significantly larger dimensions than those observed by TEM (Fig. 3) that get excluded from the thin films prepared for electron microscopy. The wormy vesicles appear extremely fluid and are aligned in one direction because of flow and also the proximity of the glass surface. (B) Phase-contrast optical micrograph of a different region of the same sample showing the elongated tubular vesicles. Bars indicate $10 \mu \mathrm{m}$. Micrographs taken with a Nikon Diaphot 300 .

senberg effect observed in the $L_{t v}$ phase under shear.

On larger length scales, we observed tubular and elongated multilamellar vesicles with optical microscopy using both differential interference contrast (DIC) (Fig. 4A) and phase contrast (Fig. 4B). Here, however, the tubules were observed on a much larger scale both in diameter $(\sim 1 \mu \mathrm{m})$ and length $(\sim 100 \mu \mathrm{m})$, although the general tubular morphologies observed seem independent of the length scale. The microscopy images show that the predominant morphology for the $L_{t v}$ phase is a dense cross-linked network of multilamellar tubular vesicles. The images reveal an abundance of conformations of tubular vesicles, most of them appearing entangled, but occasionally flow-induced alignment was observed. On rare occasions, we observed breakage of the long vesicles followed by a slow retraction of the ends. Microscopy also reveals that these cylindrical vesicles coexist with some extremely large spherical vesicles that are often highly convoluted and multilamellar. In the case of accidental flow, the vesicle structures elongated and with- 
Fig. 5. Longitudinal $x$-ray scans for a sample in the $L_{t}$ phase (triangles) (75.4 weight $\%$ water, 18.1 weight $\%$ DMPC, and 6.5 weight \% geraniol) and the $L_{\alpha}$ phase (squares) (21 weight \% water, 61 weight $\%$ DMPC, and 18 weight $\%$ geraniol). The multiple harmonics observed in the $L_{\alpha}$ phase give an interlayer spacing $d=44$ $\AA$. The narrowness and position of the peak at small wave vectors in the $L_{t v}$ phase indicates that the tubules observed in the microscopy experiments consist of multilayers with an average $d=2 \pi / q=161 \AA$. Note the expanded scale to the right.

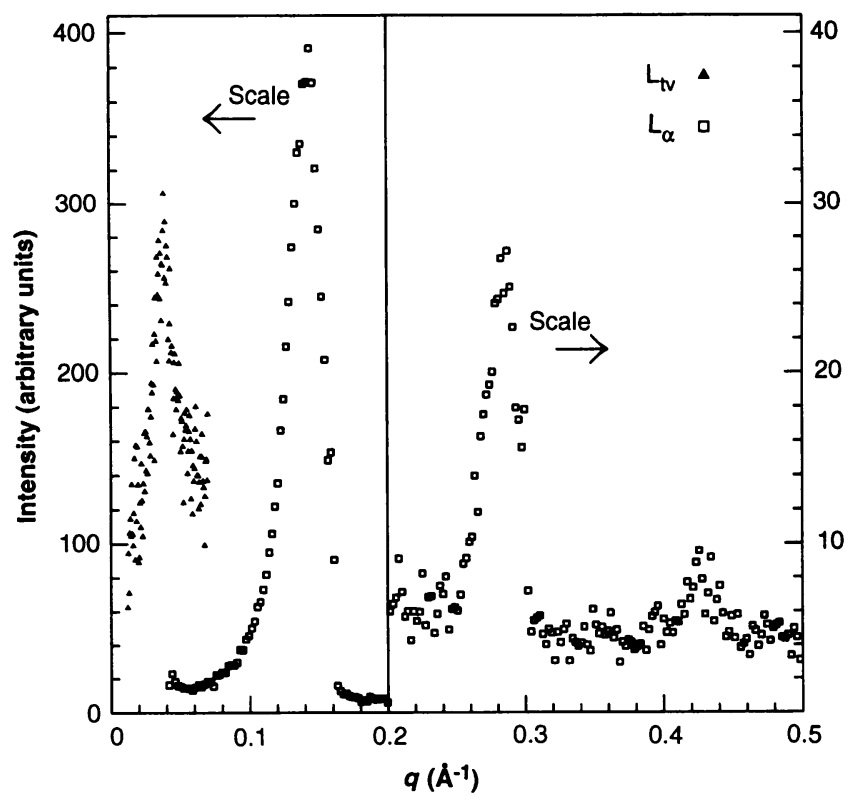

stood tension, as seen by the absence of out-of-plane fluctuations.

The similarities of the cryo-TEM and optical micrographs is striking even though the dimensions differ by almost two orders of magnitude. In thin specimens prepared for electron microscopy, vesicles larger than a few micrometers in diameter were excluded from films, and hence, only smaller vesicles were imaged. Vesicles of such size were not observed in optical microscopy images because they were beyond the resolution limit. However, the combined electron and optical micrographs show that the tubule diameters span a range from tens of nanometers to many micrometers, with their length approaching macroscopic dimensions.

In situ structural studies were carried out with $\mathrm{x}$-ray scattering on samples of the $\mathrm{L}_{\mathrm{tv}}$ and $\mathrm{L}_{\alpha}$ phases both at our in-house Materials Research Laboratory x-ray facility and, at much smaller wave vectors, on beam line VI-2 at the Stanford Synchrotron Radiation Laboratory (SSRL). The solutions were contained in sealed quartz capillaries with diameters of 1.5 and $2.0 \mathrm{~mm}$, which yielded randomly oriented lamellar domains for samples in the $L_{\alpha}$ phase. Scattering profiles from longitudinal scans for the $\mathrm{L}_{\mathrm{tv}}$ phase (triangles) and the $\mathrm{L}_{\alpha}$ phase (squares) are shown in Fig. 5. The multiple harmonics observed in the $L_{\alpha}$ phase are typical of the scattering from a low water content $L_{\alpha}$ sample; here, at 20 weight $\%$ water, they show an interlayer spacing $d=44 \AA$. At the maximum limit of water dilution for the $L_{\alpha}$ phase (46 weight \% water with 14 weight \% geraniol), $d=63 \AA$. From the sharpness and position of the peak at small wave vectors in the $\mathrm{L}_{\mathrm{tv}}$ phase (Fig. 5) combined with the microscopy images, we infer that the tubules consist of multibilayers with an average $d=2 \pi / q=161 \AA$, where $q$ is the scattering vector. The spacing between bilayers in the tubular vesicles measured from cryo-TEM images are in qualitative agreement with the value obtained from the $\mathrm{x}$-ray data.

The large average $d$ spacing measured "in situ" for this charge-neutral lipid-biological cosurfactant system in the $\mathrm{L}_{\mathrm{tv}}$ phase leads us to believe that the membrane structure in this phase of multilamellar tubular vesicles is stabilized by undulation repulsion (15-21). As was shown earlier (18), cosurfactants induce thinning of the lipid membranes, which leads to a lowering of the bending rigidity of the bilayers and stronger undulation repulsions $(27,28)$. Our observation that the maximum average interlayer spacing is significantly larger in the $\mathrm{L}_{\mathrm{tv}}$ compared with the $L_{\alpha}$ phase suggests an asymmetric partitioning with a larger cosurfactant:lipid ratio (and consequently larger undulations) in the membranes of the $\mathrm{L}_{\mathrm{tv}}$ phase. Cosurfactants are known to affect the bending rigidity $(12$, $15,18)$, but they may also affect the Gaussian rigidity of the membranes (12). For example, mixed surfactant systems can lead to the formation of anomalous isotropic phases such as the multiply connected $\mathrm{L}_{3}$ phases (13). A change in the Gaussian rigidity might lead to changes in the preferred membrane topology, leading to the stabilization of extended tubules and the spindle shapes observed in the micrographs (5).

\section{REFERENCES AND NOTES}

1. D. D. Lasic, Liposomes: From Physics to Applications (Elsevier, Amsterdam, 1993).

2. A. D. Bangham, M. W. Hill, N. G. A. Miller, Methods Membr. Biol. 1, 1 (1973).

3. See, for example, D. Papahjadjopoulos, Ed., Liposomes and Their Use in Biology, Ann. N.Y. Acad. Sci. 308, 1-412 (1978).
4. J. N. Israelachvili, Intermolecular and Surface Forces (Academic Press, London, ed. 2, 1992).

5. S. A. Safran, Statistical Thermodynamics of Surfaces, Interfaces, and Membranes (Addison-Wesley, Reading, MA, 1994).

6. See, for example, chap. 19 of (1), which contains a list of commercial cosmetics products.

7. See, for example, chap. 11 of (1).

8. E. W. Kaler, A. K. Murthy, B. E. Rodriguez, J. A. N. Zasadzinski, Science 245, 1371 (1989).

9. H. Hoffman, C. Thunig, U. Munkert, H. W. Meyer, W. Richter, Langmuir 8, 2629 (1992); G. Porte, J. Marginan, P. Basserau, R. May, Europhys. Lett. 7, 713 (1988); C. Coulon, D. Roux, A.-M. Bellocq, Phys. Rev. Lett. 66, 1709 (1991).

10. P. Hervé, D. Roux, A.-M. Bellocq, F. Nallet, T. GulikKrzywicki, J. Phys. II (France) 3, 1255 (1993).

11. B. Klösgen and W. Helfrich, Eur. Biophys. J. 22, 340 (1993); W. Helfrich and W. Habrich, Chem. Scr. 25, 32 (1985); J. Phys. (France) 51, 1027 (1990).

12. See, for example, A. Ben-Shaul, W. Gelbart, D. Roux, Eds., Micelles, Membranes, Microemulsions, and Monolayers (Springer-Verlag, New York, 1994).

13. G. Porte, J. Appell, P. Bassereau, J. Marignan, J. Phys. (France) 50, 1335 (1989); R. Strey, R. Schomäker, D. Roux, F. Nallet, U. Olsson, J. Chem. Soc. Faraday Trans. 86, 2254 (1990)

14. L. Cantu, M. Corti, M. Mussolino, P. Salina, Euro- $\infty$ phys. Lett. 13, 561 (1990).

15. C. R. Safinya et al., Phys. Rev. Lett. 57, 2718 (1986).

16. M. B. Schneider, J. T. Jenkins, W. W. Webb, J. Phys. (France) 45, 1457 (1984); Biophys. J. 45, 891 (1984). ঝ

17. W. Harbich and W. Helfrich, Chem. Phys. Lipids 36, 39 (1984); R. M. Servuss and W. Helfrich, in Physics $c$ of Complex and Supramolecular Fluids, S. A. Safran and N. A. Clark, Eds. (Wiley, New York, 1987); R. M. O) Servuss and W. Helfrich, J. Phys. (France) 50, 809 Cे (1989).

18. C. R. Safinya, E. B. Sirota, D. Roux, G. S. Smith, Phys. Rev. Lett. 62, 1134 (1989); D. Roux and C. R. Safinya, J. Phys. (France) 49, 307 (1988).

19. W. Helfrich, Z. Naturforsch. A 33, 305 (1978); W. Helfrich, J. Phys. (Paris) 47, 321 (1986).

20. E. A. Evans and V. A. Parsegian, Proc. Natl. Acad. Sci. U.S.A. 83, 7132 (1986).

21. T. J. Mclntosh, A. D. Magid, S. A. Simon, Biophys. J. 55, 897 (1989)

22. A. Tardieu, B. Luzzati, F. C. Reman, J. Mol. Biol. 75, 711 (1973); M. J. Janiak, D. M. Small, G. G. Shipley, J. Biol. Chem. 254, 6068 (1979).

23. K. Weissenberg, Nature 159, 310 (1947).

24. See, for example, R. B. Bird, R. C. Armstrong, O. Hassager, Dynamics of Polymeric Liquids (Wiley, New York, 1977), vol. 1.

25. M. E. Cates and S. J. Candau, J. Phys. Condens. Matter 2, 6869 (1990).

26. J. R. Bellare, H. T. Davis, L. E. Scriven, Y. Talmon, J. Electron Microsc. Tech. 10, 87 (1988).

27. Light-scattering experiments on the shape fluctuations of monodisperse vesicles prepared by extrusion from $L_{N}$ phase bilayers indicates that the bending modulus, $k_{c}$, is reduced to about $2 k_{B} T$ (M. Spector and J. A. Zasadzinski, in preparation).

28. Preliminary results in our laboratory show the existence of this phase in DMPC-water mixtures with other cosurfactants, such as pentanol, hexanol, and heptanol.

29. We thank P. Pincus , J. Israelachvili , E. Kaler, and D. Roux for useful comments. In particular, M. Cates is acknowledged for discussions on the Weissenberg flow effects. C.R.S., H.E.W., and S.H.J.I. gratefully acknowledge partial support by the National Science Foundation (NSF) under grant DMR-93-01199 and the Petroleum Research Fund (27837-AC7). J.A.Z., S.C., and E.N. acknowledge partial support by the National Institutes of Health under grant GM47334 and NSF under grant CTS9102719. The Materials Research Laboratory at Santa Barbara is supported by NSF under grant DMR-9123048. The synchrotron $x$-ray scattering experiments were carried out on beam line $\mathrm{VI}-2$ at the SSRL, which is supported by the U.S. Department of Energy.

21 June 1994; accepted 13 September 1994 